\title{
Resonance zones for interactions of magnetosonic waves with radiation belt electrons and protons
}

\author{
Wenxun Zhang, Ruoxian Zhou, Juan Yi, Xudong Gu*, Binbin Ni, Chengyao Zheng and Man Hua
}

\begin{abstract}
As an important plasma wave mode in the geospace, magnetosonic waves can interact with both radiation belt electrons and protons, thereby impacting the dynamics of magnetospheric particles. Based on the Doppler-shifted resonance condition and the cold plasma dispersion relation, we investigate the profiles of resonance zone and resonant frequency of the Landau resonance between radiation belt electrons and magnetosonic waves and the cyclotron resonances with protons. The results demonstrate that resonant interactions between magnetosonic waves and magnetospheric charged particles largely rely on L-shell, wave normal angle, and kinetic energy and equatorial pitch angle of particles. Resonance zones for the Landau resonance between magnetosonic waves and radiation belt electrons are confined to a very narrow (mostly less than $1^{\circ}$ ) extent of magnetic latitude, which tends to shift to lower latitudes with increasing equatorial pitch angle and decreasing electron energy. Landau resonance frequencies also increase with magnetosonic wave normal angle. In contrast, higher order cyclotron resonances of magnetosonic waves with protons are much easier to occur in a broad range of magnetic latitude. As the resonance order increases, the coverage of the resonance zone shrinks overall and occupies the geomagnetic equatorial region. In addition, resonant frequencies increase with resonance order. Corresponding to higher order cyclotron resonances, protons are more likely to interact with magnetosonic waves at intermediate to high frequencies. Our study can be useful to elaborate the resonant interaction processes between magnetosonic waves and radiation belt electrons and protons and improve the current understanding of the multi-aspect impact of magnetosonic waves on the magnetospheric particle dynamics.
\end{abstract}

Keywords: Magnetosonic waves, Radiation belts, Wave-particle interactions, Resonance zone and resonant frequency

\section{Background}

Magnetosonic waves in the geospace, also known as equatorial noises, are an important space plasma wave mode (Russell et al. 1970). Numerous observations have shown that the frequencies of magnetosonic waves are generally between the proton cyclotron frequency and the lower hybrid resonant frequency. They propagate almost perpendicular to the background magnetic field. Magnetosonic waves are found to exist both inside and outside the plasmasphere and occur characteristically in

\footnotetext{
*Correspondence: guxudong@whu.edu.cn

Department of Space Physics, School of Electronic Information, Wuhan University, Wuhan 430072, Hubei, China
}

the vicinity of the magnetic equator (Russell et al. 1970; Laakso et al. 1990; Santolík et al. 2002; Ma et al. 2013) and also occasionally extend to higher latitudes up to $\sim 60^{\circ}$ (Tsurutani et al. 2014).

In recent years, further studies have shown that space plasma waves, including magnetosonic waves, can significantly affect the dynamics of the magnetospheric particles (e.g., Horne et al. 2007; Xie et al. 2007; Li et al. 2008; Gu et al. 2011; Chang et al. 2013; Xiao et al. 2015). Based on the Cluster wave observations, Horne et al. (2007) established a model of magnetosonic waves for adoption to compute their quasi-linear bounce-averaged diffusion coefficients. Their results indicated that the Landau resonance caused by magnetosonic waves is an

\section{Springer Open}

(c) The Author(s) 2017. This article is distributed under the terms of the Creative Commons Attribution 4.0 International License (http://creativecommons.org/licenses/by/4.0/), which permits unrestricted use, distribution, and reproduction in any medium, provided you give appropriate credit to the original author(s) and the source, provide a link to the Creative Commons license, and indicate if changes were made. 
important potential mechanism to accelerate the radiation belt electrons. Subsequent studies (Mourenas et al. 2013) developed a simplified formula to calculate the quasi-linear diffusion coefficients of radiation belt electrons by magnetosonic waves. They also reported that the pitch angle scattering effect of magnetosonic waves is weak for electron precipitation losses, but the momentum diffusion is relatively strong for electron energization. Xiao et al. (2015) found that the combined effect of magnetosonic waves and chorus waves with radiation belt electrons can lead to the diffusion of electrons from high pitch angles of $\sim 90^{\circ}$ to intermediate pitch angles, resulting in "butterfly-like" electron distributions (Gannon et al. 2007). The significance of Landau resonant scattering by equatorial magnetosonic waves has been further recognized to account for the formation of energetic electron butterfly distributions in the inner magnetosphere from the outer edge of the inner zone to much higher L-shells (e.g., Li et al. 2016a, b; Ma et al. 2016). In addition, magnetosonic wave-induced bounce resonance has been proposed in terms of test particle simulation as a responsible mechanism for the rapid modulation of butterfly distributions (Maldonado et al. 2016). All of the above work focused on the interaction between magnetosonic waves and radiation belt electrons. In contrast, there was little work about the interaction processes between magnetosonic waves and protons. A recent study by Xiao et al. (2014) explored the potential contribution of magnetosonic wave scattering to the generation of the proton aurora. Our study clearly demonstrates that radiation belt electrons and protons can undergo resonant interactions with MS waves at the latitudes away from the geomagnetic equator, distinguishing it from most previous studies that focus on MS waves at the equatorial region.

In order to study in detail the interactions between magnetosonic waves and radiation belt electrons and protons, the present study will calculate the resonant frequencies for the interactions between magnetosonic waves and magnetospheric particles, and systematically analyze the corresponding resonance zones to which latitudinal extent resonant wave-particle interactions can take place. "Magnetosonic wave dispersion relation and wave-particle resonance condition" will briefly introduce the dispersion relation of magnetosonic waves and the general resonance condition of wave-particle interactions. "Resonance zones for radiation belt electrons interacting with magnetosonic waves" and "Resonance zones for protons interacting with magnetosonic waves" will explore the resonant frequencies and resonance zones of magnetosonic waves interacting with radiation belt electrons and protons, respectively, followed by our conclusions at the end.

\section{Magnetosonic wave dispersion relation and wave- particle resonance condition}

According to the classical cold plasma theory of wave propagation in the magnetized plasma (Stix 1962), the general equation that describes the dispersion relation of space plasma wave propagation follows:

$$
A n_{\mathrm{r}}^{4}-B n_{\mathrm{r}}^{2}+C=0,
$$

where $n_{\mathrm{r}}=(k c) / \omega$ is the refractive index, $k$ is the wave number, $c$ is the speed of light in the vacuum, and $\omega$ is the wave angular frequency. The polynomial equation coefficients are as follows: $A=S x^{2}+P$, $B=R L x^{2}+P S\left(2+x^{2}\right), C=P R L\left(1+x^{2}\right), x=\tan \theta$ with $\theta$ as the wave normal angle. In addition, $P, R, L$, and $S$ are Stix parameters, which can be expressed as follows:

$$
\begin{aligned}
& P=1-\frac{\omega_{p i}^{2}}{\omega^{2}}-\frac{\omega_{p e}^{2}}{\omega^{2}}, \\
& R=1-\frac{\omega_{p i}^{2}}{\omega\left(\omega+\Omega_{c i}\right)}-\frac{\omega_{p e}^{2}}{\omega\left(\omega-\Omega_{c e}\right)}, \\
& L=1-\frac{\omega_{\mathrm{pi}}^{2}}{\omega\left(\omega-\Omega_{\mathrm{ci}}\right)}-\frac{\omega_{\mathrm{pe}}^{2}}{\omega\left(\omega+\Omega_{\mathrm{ce}}\right)}, \\
& S=\frac{R+L}{2},
\end{aligned}
$$

where $\quad \Omega_{c i}=e\left|B_{0}\right| / m_{i} \quad$ and $\quad \Omega_{c e}=e\left|B_{0}\right| / m_{e} \quad$ are, respectively, the electron and ion gyrofrequencies, $\omega_{p i}=\left(N_{0} e^{2} / \varepsilon_{0} m_{i}\right)^{1 / 2}$ and $\omega_{p e}=\left(N_{0} e^{2} / \varepsilon_{0} m_{e}\right)^{1 / 2}$ are, respectively, the ion and electron plasma frequencies, $e$ is the unit charge, $\varepsilon_{0}$ is the dielectric constant in the vacuum, $m_{i}$ and $m_{e}$ are, respectively, the rest mass of protons and electrons, $B_{0}$ is the local geomagnetic field strength, and $N_{0}$ is the electron number density. In this study, we assume that hydrogen ions (i.e., protons) are the only ion population. According to the charge neutrality, the proton density is equal to the electron density.

According to the above cold plasma dispersion relation, we take into account the occurrence of magnetosonic waves both inside and outside the plasmasphere. Therefore, this study focuses on the analysis of magnetosonic wave dispersion at two representative spatial locations, say $L=3$ in the plasmasphere and $L=6$ in the plasma trough. Firstly, the ambient magnetic field is assumed as dipolar, i.e.,

$$
\begin{aligned}
& B_{0}=B_{\text {eq }} f(\lambda)(\mathrm{nT}), \\
& f(\lambda)=\frac{\left(1+3 \sin ^{2}\right)^{1 / 2}}{\cos ^{6} \lambda} .
\end{aligned}
$$


In Eq. (6), $B_{\text {eq }}=M / L^{3}$ is the equatorial geomagnetic field strength at the Earth's surface and $M=30911.553 \mathrm{nT} \cdot R_{e}$ is the magnetic dipole moment.

In addition, by assuming the plasmapause location at $L_{\mathrm{pp}}=3.5$, we use the empirical plasma density model of Sheeley et al. (2001):

In the plasmasphere $\left(L<L_{\mathrm{pp}}\right)$ :

$$
N_{\text {eq }}=1390\left(\frac{3}{L}\right)^{4.83}\left(\mathrm{~cm}^{-3}\right) \text {. }
$$

In the plasma trough $\left(L>L_{\mathrm{pp}}\right)$ :

$$
N_{\mathrm{eq}}=124\left(\frac{3}{L}\right)^{4.0}\left(\mathrm{~cm}^{-3}\right) .
$$

Using the models above, we can calculate the dispersion relation of magnetosonic waves. Figure 1 shows the dispersion relation $(\omega, k)$ curves for magnetosonic waves propagating at the wave normal angles of $87^{\circ}$ and $89^{\circ}$ at the geomagnetic equator $\left(\lambda=0^{\circ}\right)$ at the spatial locations of $L=3$ and $L=6$. Following previous studies
(Horne et al. 2007; Li et al. 2014; Chen et al. 2015), magnetosonic wave frequencies $(\omega)$ are considered within the range $0.0026<\omega /\left(\Omega_{c e}\right)_{\mathrm{eq}}<0.0044$, where $\left(\Omega_{c e}\right)_{\mathrm{eq}}$ is the electron gyrofrequency at the geomagnetic equator. The frequency interval is indicated by two red vertical lines in Fig. 1. The adopted parameters are also given in the subplots. It can be seen from Fig. 1 that the wave normal angle has little effect on the dispersion curve of magnetosonic waves. But given the normalized wave frequency of magnetosonic waves, the phase velocity and group velocity are smaller at $L=3$ in the plasmasphere than at $L=6$ outside the plasmasphere.

The Doppler-shifted resonance condition between charged particles and plasma waves in the geospace satisfies

$$
\omega-k c \beta \cos \theta \cos \alpha=n \Omega_{c \sigma} / \gamma,
$$

where $\alpha$ is the particle pitch angle, $\gamma=\left(1-\beta^{2}\right)^{-1 / 2}$ is the relativistic factor, $\beta=v / c, v$ is the particle thermal velocity, $\Omega_{c \sigma}$ is the non-relativistic particle gyrofrequency, and $N$ is an integer as resonant harmonic number with $N=0$ for the Landau resonance.
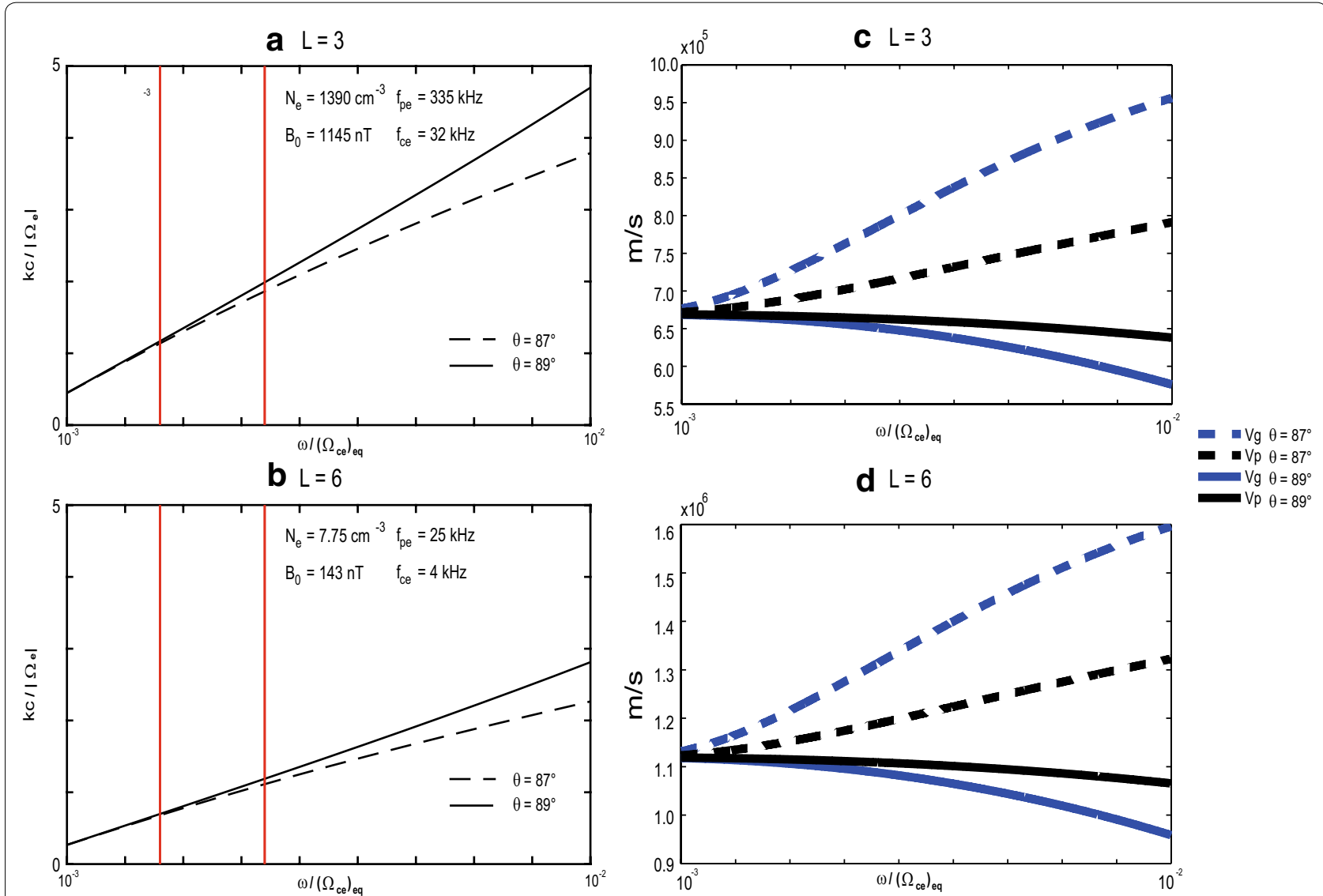

Fig. 1 Dispersion relation curves of equatorial magnetosonic waves at the wave normal angles of 87 and 89 : $\mathbf{a}$ inside the plasmasphere at $L=3$ and $\mathbf{b}$ outside the plasmasphere at $L=6$. $\mathbf{c}$ Wave phase velocity and $\mathbf{d}$ group velocity 
According to the conservation of the first adiabatic invariant, the local pitch angle $\alpha$ along the geomagnetic line satisfies

$$
\sin \alpha=\sin \alpha_{\text {eq }}[f(\lambda)]^{1 / 2} .
$$

where $\alpha_{\mathrm{eq}}$ is the equatorial pitch angle. Whether charged particles can resonantly interact with magnetospheric waves is determined simultaneously by Eqs. (1) and (10). By combining these two equations, we can obtain a 10thorder polynomial equation about resonant frequency $\omega$ as follows:

$$
\begin{gathered}
A_{10} \omega^{10}+A_{9} \omega^{9}+A_{8} \omega^{8}+A_{7} \omega^{7}+A_{6} \omega^{6}+A_{5} \omega^{5} \\
+A_{4} \omega^{4}+A_{3} \omega^{3}+A_{2} \omega^{2}+A_{1} \omega+A_{0}=0,
\end{gathered}
$$

where $A_{0}-A_{10}$ are the coefficients of the polynomial equation, the exact expressions of which are given in Appendix.

Given the spatial position (determined by the $L$ value and the geomagnetic latitude), the background magnetic field and the electron density can be obtained. Other parameters, including particle energy, pitch angle, resonant harmonic number and wave normal angle, are used to resolve Eq. (12) for solutions resonant wave frequency. If the obtained wave frequency $\omega$ is within the given frequency band of magnetosonic waves, it is considered that the resonant interaction occurs; otherwise, there is no resonance between the waves and the particles at the specific location. In this manner, a comprehensive analysis can be performed to investigate the resonant frequencies and resonance zones between magnetosonic waves and magnetospheric particles and distinguish the related resonance properties. Here the term 'resonance zone' denotes the latitudinal extent of magnetosonic waves that can undergo resonant interactions with magnetospheric particles, that is, satisfying the Dopplershifted resonance condition $(\omega-k\|v\|=N \Omega / \gamma$, where $N=0, \pm 1, \pm 2, \ldots)$.

\section{Resonance zones for radiation belt electrons interacting with magnetosonic waves}

We first analyze the resonance zones between magnetosonic waves and radiation belt electrons, in particular the Landau resonance zone. The Landau resonance zone denotes the latitudinal extent of MS waves that can undergo the Landau resonance with magnetospheric particles, that is, satisfying the Landau resonance condition $(\omega=k|| v||)$. When the background magnetic field line is given, equatorial radiation belt energetic electrons with different pitch angles bounce forth and back along the geomagnetic field line between the northern and southern hemispheres, thereby encountering magnetosonic waves in space. Once the resonance condition
(Eq. (10)) is satisfied, the waves and electrons resonate with each other and the energy is transferred between them. By solving Eq. (12), we can determine the useful resonant frequencies that lie within the wave frequency spectrum. Here we consider the resonance zones for six specific electron energies: $30 \mathrm{keV}, 100 \mathrm{keV}, 300 \mathrm{keV}$, $1 \mathrm{MeV}, 3 \mathrm{MeV}$, and $10 \mathrm{MeV}$, to cover nearly three orders of energy from low-energy electrons to ultra-relativistic electrons. In order to quantitatively analyze the effect of wave normal angle on the extent of resonance zones, we choose the two cases of $\theta=87^{\circ}$ and $\theta=89^{\circ}$.

Figure 2 shows the variations of resonant frequency in (equatorial pitch angle $\alpha_{\mathrm{eq}}$, magnetic latitude $\lambda$ ) space for the Landau resonance between magnetosonic waves and radiation belt electrons at $L=3$ inside the plasmasphere. The wave frequency band is adopted as $0.0026<\omega /\left(\Omega_{c e}\right)_{\mathrm{eq}}<0.0044$, corresponding to a frequency range of 83-141 $\mathrm{Hz}$. When the calculated resonant frequency, as color coded, is within this range, it

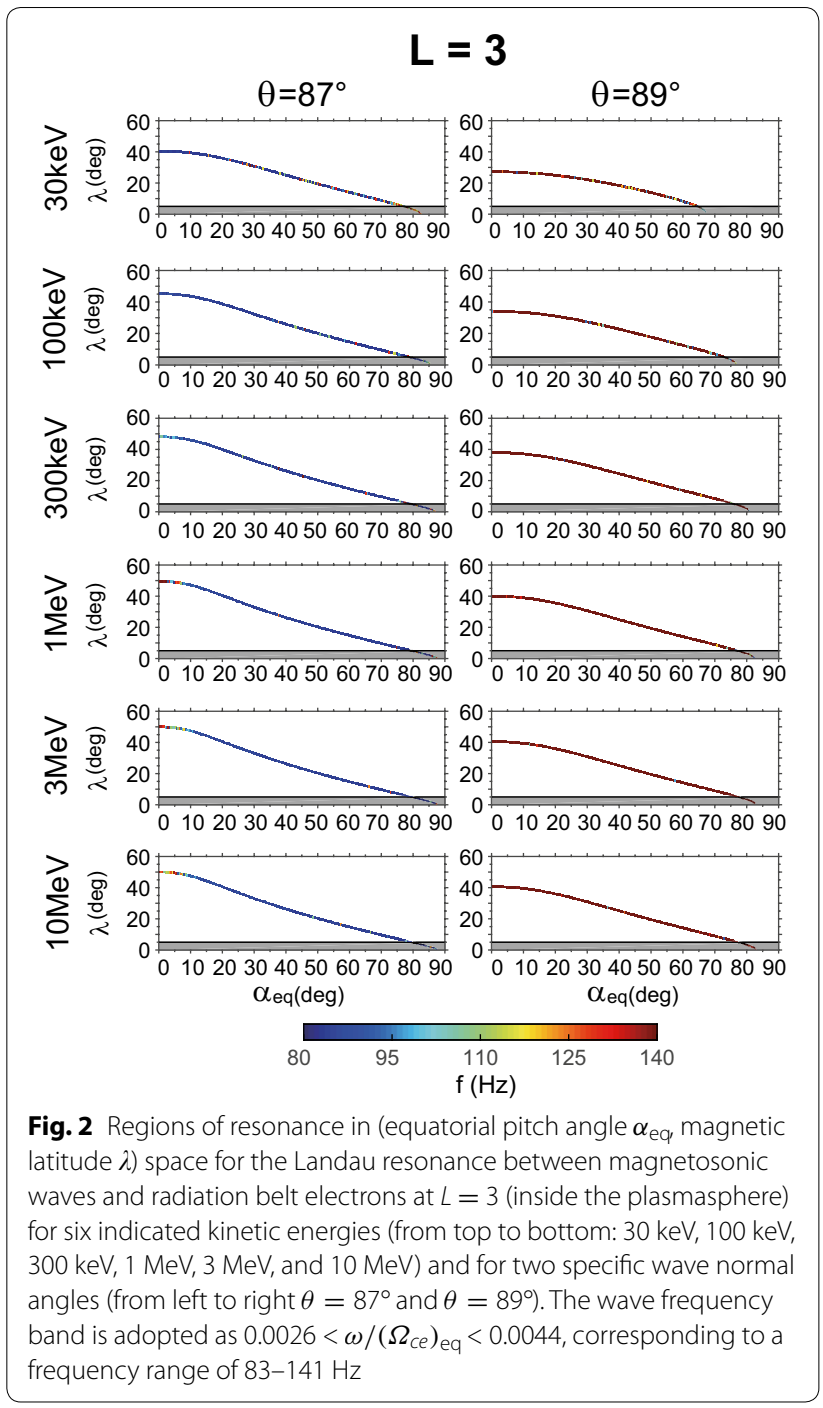


means that the Landau resonance can occur. In each subplot, the horizontal black solid line marks the magnetic latitude of $5^{\circ}$ below which magnetosonic waves are assumed to be commonly present. The shaded regions under the black line indicate the dominant area of resonant wave-particle interactions. Resonance zones for radiation belt electrons are more concentrated in the line shown, and the other areas are weaker.

It can be observed from Fig. 2 that the resonance zones along the geomagnetic field lines demonstrate a narrow region for considered electron energies and wave normal angles. A further check confirms that the latitudinal extent of the Landau resonance zone between magnetosonic waves and radiation belt electrons is no more than $1^{\circ}$.

Given electron energy, the resonance zones gradually decrease with equatorial pitch angle. It is seen that for small pitch angle close to $0^{\circ}, 30 \mathrm{keV}$ electrons can resonate with the waves at latitudes of $\sim 40^{\circ}$, while they can only resonate with the equatorial waves for high pitch angles of $\sim 90^{\circ}$, mainly owing to the mirroring geometry of electrons. Regarding the resonant wave frequencies, magnetosonic waves tend to resonate with electrons at frequencies of the lower frequency portion of the wave spectrum, which show only small variations. But for high electron energies (i.e., $\geq 1 \mathrm{MeV}$ ), resonant frequencies lie at the high-frequency side of the wave spectrum for $<\sim 5^{\circ}$ equatorial pitch angles. As the equatorial pitch angle increases, they shift quickly to the low-frequency side, suggesting considerable changes in the Landau resonance condition between magnetosonic waves and radiation belt electrons at different pitch angles.

Given the equatorial pitch angle, the magnetic latitudes of resonance zone elevate as the electron energy increases. Specifically, at small pitch angles, magnetosonic waves with $\theta=87^{\circ}$ can resonate with $30 \mathrm{keV}$ electrons at the latitudes of $\sim 40^{\circ}$ but resonate with $10 \mathrm{MeV}$ electrons near $50^{\circ}$ latitudes. In contrast, the resonant wave frequencies vary insignificantly with electron energy. For the case of $\theta=89^{\circ}$, the resonant frequencies are commonly high, approaching the upper limit of magnetosonic wave frequency spectrum adopted in this study, regardless of electron energy.

In addition, the wave normal angle has a strong effect on the determination of resonance zone and resonant frequency. In general, larger wave normal angles produce resonance zones at lower latitudes. In the vicinity of loss cone $\left(\sim 8^{\circ}\right), 30 \mathrm{keV}$ electrons resonate with magnetosonic waves with $\theta=87^{\circ}$ at the magnetic latitudes of $\sim 40^{\circ}$ but resonate with the waves with $\theta=89^{\circ}$ at $\sim 27^{\circ}$ latitudes. $1 \mathrm{MeV}$ electrons interact resonantly with magnetosonic waves with the above two normal angles at the magnetic latitudes of around $50^{\circ}$ and $40^{\circ}$, respectively. A larger electron population that covers a broader range of

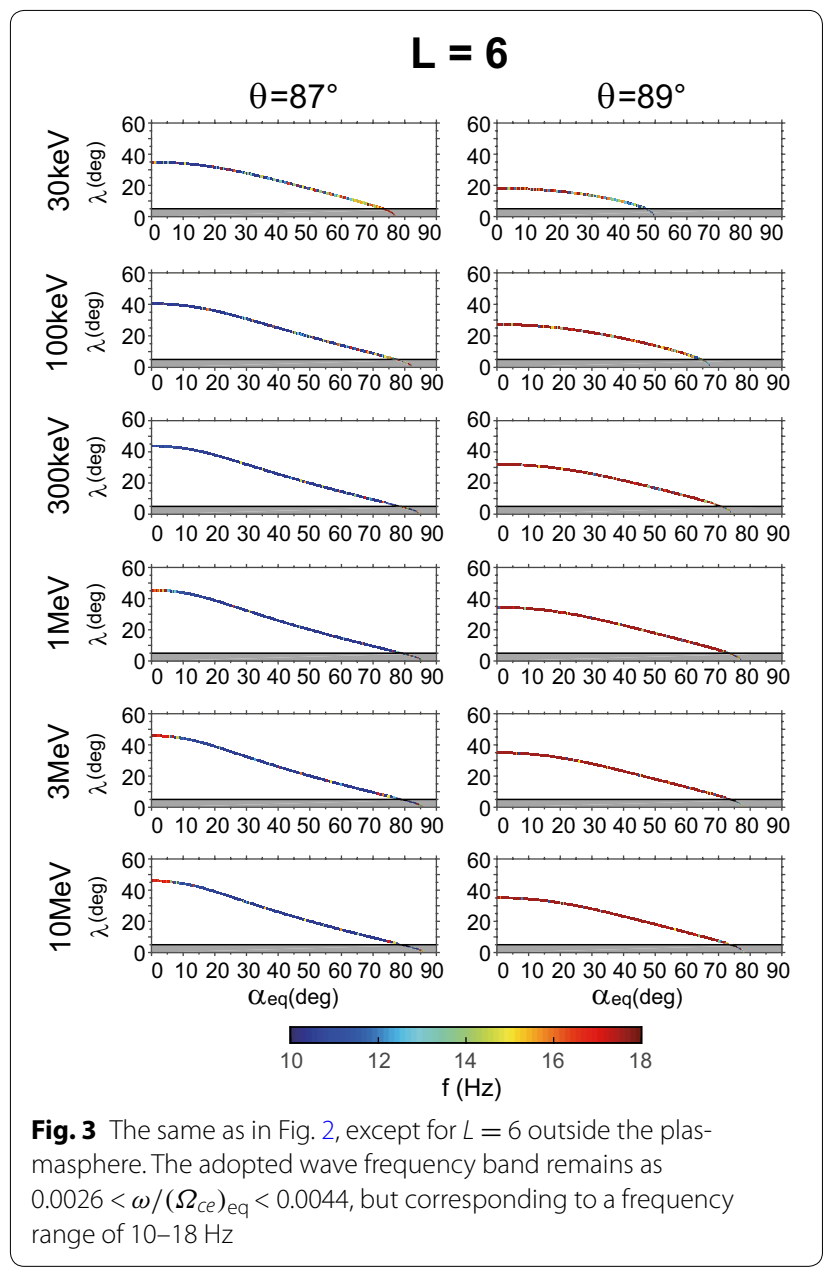

equatorial pitch angle can resonate with magnetosonic waves with $\theta=87^{\circ}$. Wave normal angle is also an important factor affecting resonant frequencies. For $300 \mathrm{keV}$ electrons, the resonant frequencies for $\theta=87^{\circ}$ are close to the low-frequency side of the selected wave spectrum, while they tend to approach the high-frequency end for $\theta=89^{\circ}$.

Similar to Figs. 2 and 3 shows the spatial Landau resonance regions of radiation belt electrons with magnetosonic waves except for $L=6$ outside the plasmasphere for the two cases of $\theta=87^{\circ}$ and $\theta=89^{\circ}$. At this spatial location, the corresponding magnetosonic wave frequency range is $10-18 \mathrm{~Hz}$. Obviously, both resonance zones of radiation belt electrons and resonant frequencies of magnetosonic waves change with the wave normal angle, magnetic latitude, and electron energy and equatorial pitch angle, following a similar trend consistent with those for $L=3$ inside the plasmasphere.

However, there still exist differences between Figs. 2 and 3. Firstly, the extents of resonance zone are different under the situation inside and outside the plasmasphere. 
For instance, for a small equatorial pitch angle and magnetonic waves with $\theta=87^{\circ}$, the Landau resonance zones of $30 \mathrm{keV}$ electrons are located near the magnetic latitude of $40^{\circ}$ for $L=3$, but around $35^{\circ}$ for $L=6$. Secondly, under the same circumstance, radiation belt electrons resonate with magnetosonic waves at smaller pitch angle at $L=3$ than at $L=6$. Thirdly, while the resonant frequencies for interactions both inside and outside the plasmasphere exhibit similar overall variation features, their respective absolute values are significantly different. It is worthwhile to note that due to the observational confinement of magnetosonic waves to the equatorial region, the shaded regions below the horizontal black solid line in each subplot are the favorable areas where the Landau resonance between magnetosonic waves and radiation belt electrons can occur.

For the cases of cyclotron resonances (i.e., $n \neq 0$ ), we also perform the computations. While the results are not shown, we point out that the electron energy should be extremely high (e.g., $>10 \mathrm{MeV}$ ) in order to undergo cyclotron resonance with magnetosonic waves (e.g., Horne et al. 2007), which however is beyond the interest of this investigation. This also justifies our concentration on the Landau resonance, which has been regarded as a dominant mechanism responsible for the interactions between magnetosonic waves and radiation belt electrons.

\section{Resonance zones for protons interacting with magnetosonic waves}

We also analyze the resonance zones between magnetosonic waves and protons that are generally below $1 \mathrm{MeV}$. Similar to radiation belt electrons, protons with different pitch angles bounce forth and back along the geomagnetic field line and encounter magnetosonic waves in space. Once the resonance condition (Eq. (10)) is satisfied, the waves and protons resonate with each other. We also follow the above similar way to compute the resonant frequencies and resonance zones for protons. We consider the resonance zones for six specific proton energies: $30 \mathrm{keV}, 100 \mathrm{keV}, 300 \mathrm{keV}, 1 \mathrm{MeV}, 3 \mathrm{MeV}$, and $10 \mathrm{MeV}$. We also choose two cases for magnetosonic wave normal angles of $\theta=87^{\circ}$ and $\theta=89^{\circ}$. Besides taking into account the resonance inside and outside the plasmasphere (i.e., $L=3$ and $L=6$ ), we analyze the resonant wave-particle interactions between magnetosonic waves and protons at different resonance orders (i.e., $n$ values). With a careful check, we find that the resonances at $n=4,5$, and 6 dominate the resonant interactions between magnetosonic waves and protons. This is consistent with the study of Fu et al. (2016), which found that the dominant scattering occurs between magnetosonic waves and protons when the resonance harmonics are close to the multiple of proton gyrofrequency covered by the wave frequency spectrum.

Figure 4 shows the resonance zones and resonant frequencies for the interactions between proton and magnetosonic waves for different energy protons at the cyclotron resonance harmonic $n=4$, corresponding to two locations of $L=3$ inside the plasmasphere and $L=6$ inside the plasma trough and two wave normal angles of $\theta=87^{\circ}$ and $\theta=89^{\circ}$. Clearly, the proton resonance zones are confined to the magnetic latitudes below $25^{\circ}$. Distinct from the results for radiation belt electrons, protons can resonate with magnetosonic waves in a much broader range of latitude. Moreover, the variations of resonance zone with proton energy and pitch angle are very distinguishable. For instance, inside the plasmasphere $(L=3)$, $300 \mathrm{keV}$ protons interact with magnetosonic waves with $\theta=87^{\circ}$ at equatorial pitch angles in the range of $27^{\circ}-74^{\circ}$, but $1 \mathrm{MeV}$ protons at $45^{\circ}-82^{\circ}$. In addition, resonant wave frequencies vary largely with proton energy and resonant magnetic latitude. For lower energy protons, the resonant frequencies mainly increase with magnetic latitude, gradually approaching the upper limit of the wave spectrum. But for high-energy (e.g., $1 \mathrm{MeV}$ ) protons and $\theta=87^{\circ}$, resonant frequencies become smaller at higher latitudes. By considering the major occurrence regions of magnetosonic waves, i.e., within a few degrees of the geomagnetic equator, higher energy protons are more likely to undergo cyclotron resonance with magnetosonic waves. When the wave normal angle changes, the resonance zones and resonant frequencies do not change much for lower energy protons, i.e., 3-10 keV, but vary significantly for higher energies. For instance, for $1 \mathrm{MeV}$ protons, they resonate with $\theta=87^{\circ}$ and $\theta=89^{\circ}$ magnetosonic waves at the equatorial pitch angles of $45^{\circ}-82^{\circ}$ and $0^{\circ}-66^{\circ}$, respectively. We also note that the spatial location is another important factor affecting the wave-particle interactions. Take the results of $300 \mathrm{keV}$ protons as an example. Their resonance zones with $\theta=89^{\circ}$ magnetosonic waves cover the equatorial region at $L=3$ but well above $5^{\circ}$ at $L=6$, which suggests that no realistic resonance can occur for the latter case when the equatorial wave confinement is taken into account.

Figure 5 displays the results for the resonance harmonic $n=5$. In general, the resonance zones and resonant frequencies show variation features with proton energy, pitch angle, wave normal angle, and L-shell in a manner similar to Fig. 4. However, we can still capture some differences. For the resonance order $n=5$, the resonance zones can cover well the equatorial region in all considered cases. The proton population with a broader range of equatorial pitch angle also gets into resonance with magnetosonic waves. In addition, the resonant 


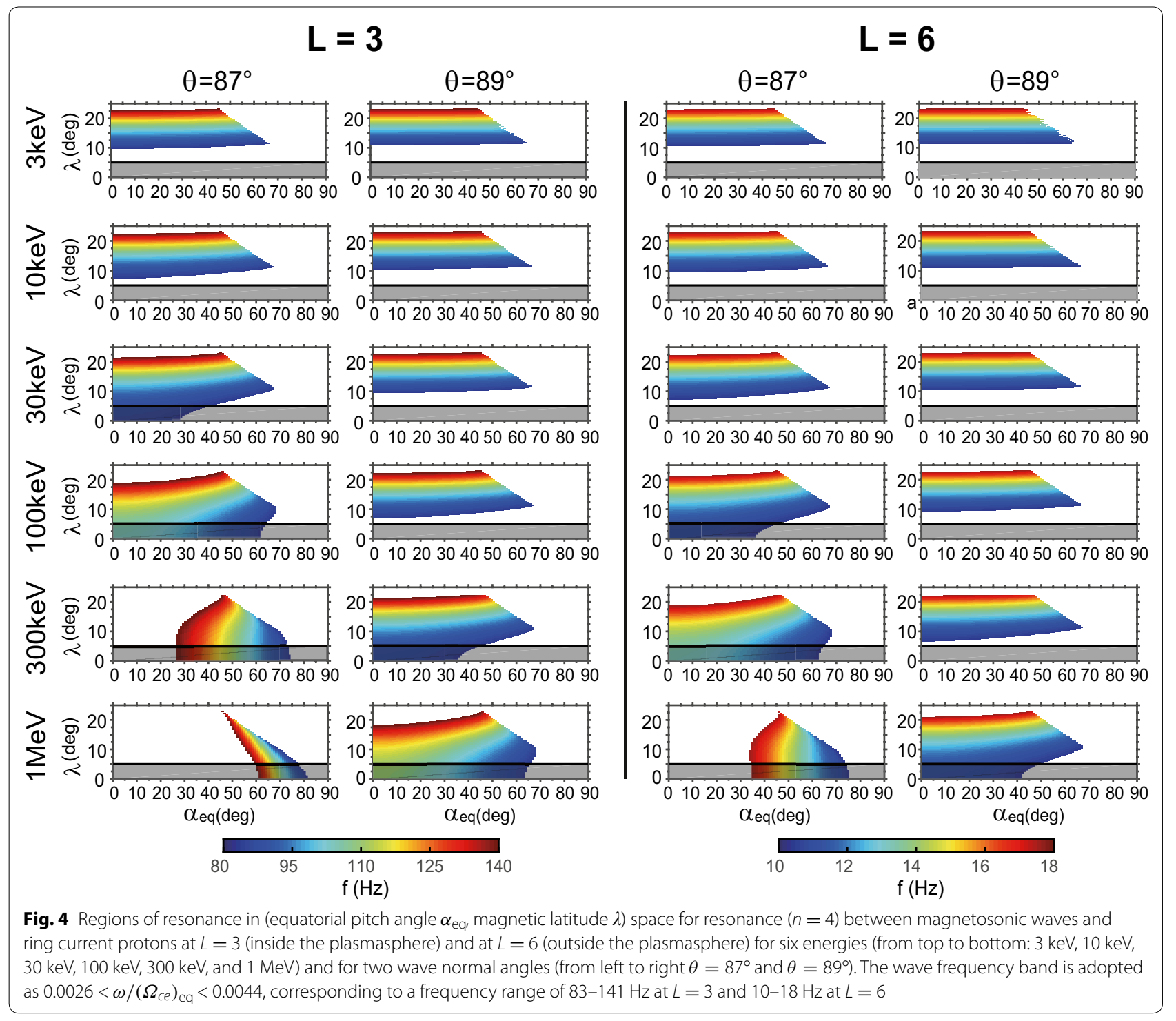

frequencies are overall higher for $n=5$ than the case of $n=4$. The results for $n=6$ are shown in Fig. 6. Notably, the variations of resonance zones and resonant frequencies are more pronounced. The latitudinal extent of resonant zone continues to shrink overall besides covering the equatorial region. Meanwhile, the resonant frequencies of $n=6$ are obviously located at the intermediate frequency and high-frequency portion of magnetosonic waves.

We also compute the results for other resonance harmonics (i.e., $n \neq 4,5,6$ ). While those results are not shown, it is worthwhile to point out that, compared to the cases of $n=4,5$, and 6 , cyclotron resonances occurring at other resonance orders are less important for the spectral distribution of magnetosonic waves selected in this study and are therefore ignored accordingly. We note that the dominant cyclotron resonance harmonics for magnetosonic waves interacting with protons can vary corresponding to changes in the wave frequency spectrum.

It is worthwhile to point out that while it is uncommon for MS waves to be present at latitudes above $10^{\circ}$, such MS waves can indeed occur at higher latitudes. The statistical analysis of Tsuratani et al. (2014), on the basis of a survey of Polar observations, reported that MS wave occurrence (and intensities) peaked within $\sim \pm 5^{\circ}$ of the magnetic equator, with half maxima at $\sim \pm 10^{\circ}$, and that MS waves can also be detected as far from the equator as $+20^{\circ}$ and $60^{\circ}$ magnetic latitude but with lower intensities. While MS waves are hardly generated at these high latitudes, their propagation effect can shed light on the observations of MS waves quite away 


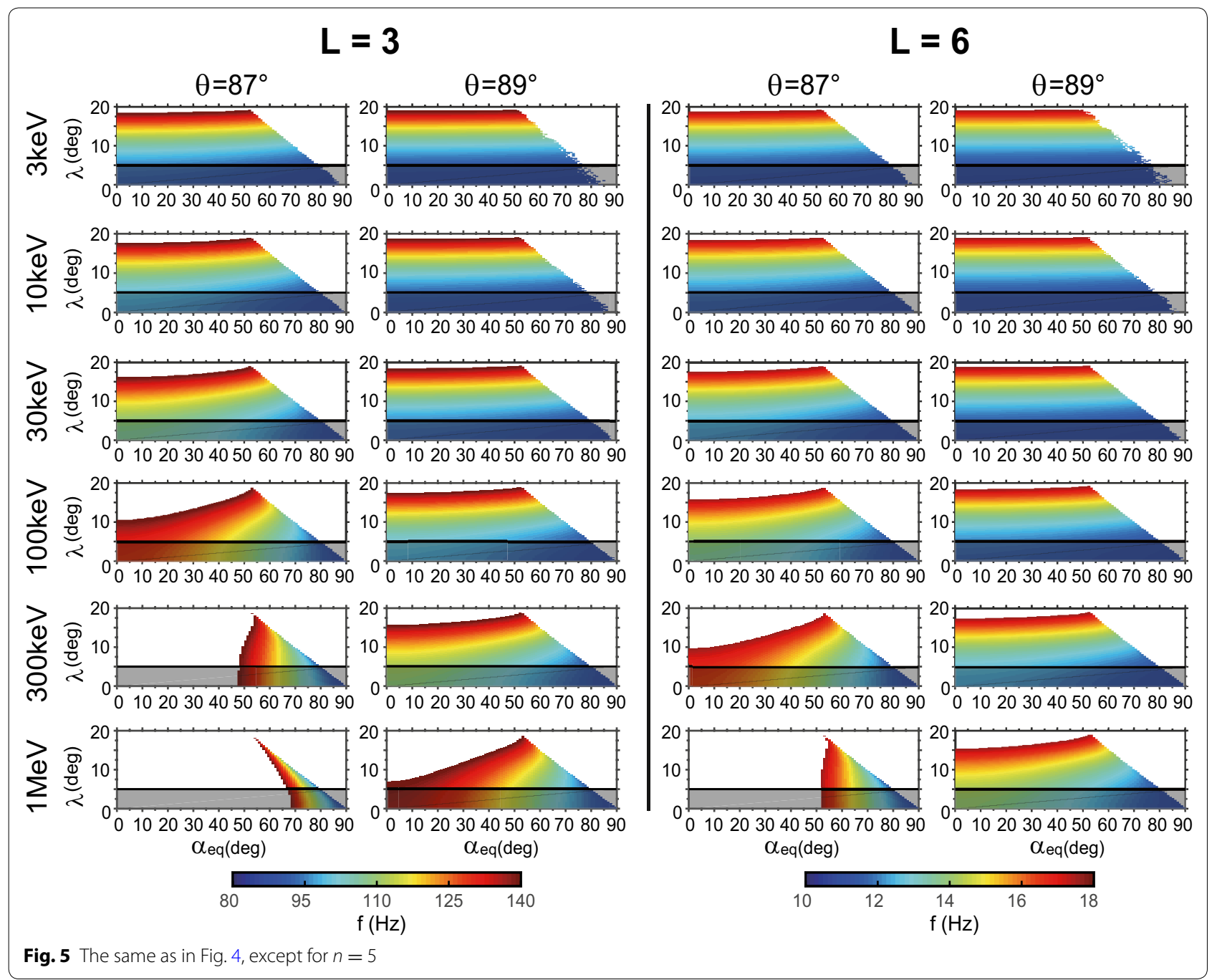

from the geomagnetic equator, e.g., as investigated in a recent study by Zhima et al. (2015). While the present study has no intention to emphasize on the possibility of high-latitude MS waves, it aims to take into account all the possible occurrence cases of MS waves, i.e., both equatorial and off-equator emissions, to understand the underlying resonant interactions between MS waves and magnetospheric particles. As a consequence, our present investigation can help improve the current understanding in this regard by incorporating the potential effect of off-equator MS waves on the magnetospheric particle dynamics from the perspective of resonant wave-particle interactions.

\section{Conclusions}

MS wave in the geospace is an important space plasma wave mode. The interactions between magnetosonic waves and radiation belt electrons and protons affect the space radiation environment. Based on the
Doppler-shifted resonance condition and cold plasma dispersion relation, we analyze the resonance zones and resonant frequency between magnetosonic waves and radiation belt electrons and protons. The results show that the interactions between magnetosonic waves and magnetospheric particles largely rely on L-shell, wave normal angle, and kinetic energy and equatorial pitch angle of particles. Resonance zones for the Landau resonance between magnetosonic waves and radiation belt electrons are confined to a very narrow (mostly less than $1^{\circ}$ ) extent of magnetic latitude, which tends to shift to lower latitudes with increasing equatorial pitch angle and decreasing electron energy. Landau resonance frequencies also increase with magnetosonic wave normal angle. By comparison, higher order cyclotron resonances of magnetosonic waves with protons are much easier to occur in a broad range of magnetic latitude. As the resonance order increases, the coverage of the resonance zone shrinks overall and occupies the 

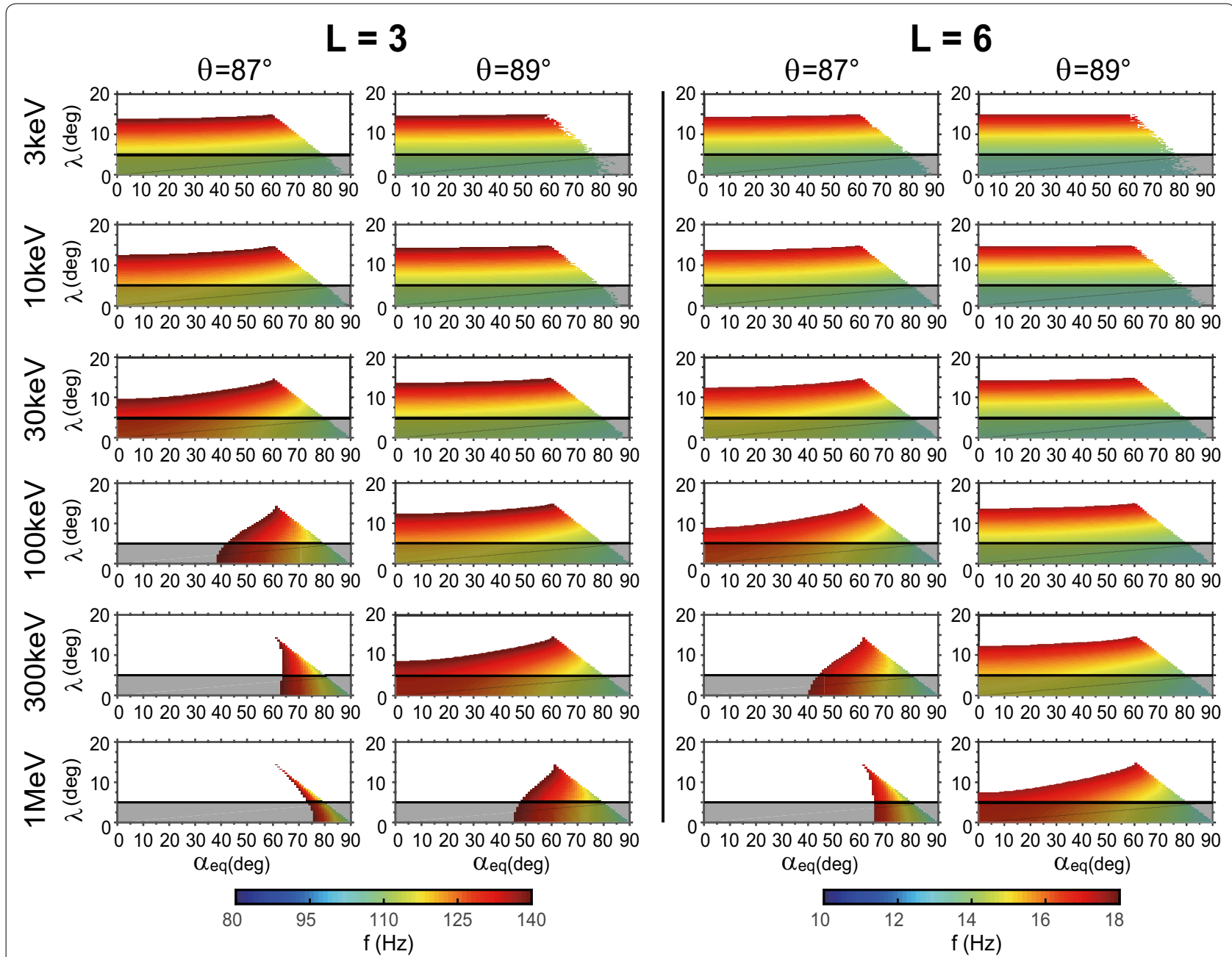

Fig. 6 The same as in Fig. 4, except for $n=6$

geomagnetically equatorial region. In addition, resonant frequencies increase with resonance order. Corresponding to higher order cyclotron resonances, protons are more likely to interact with magnetosonic waves at intermediate to high frequencies. Our study can be a useful addition to the current literature to elaborate the interaction processes between magnetosonic waves and radiation belt electrons and protons, including Landau resonance, cyclotron resonance, transit-time scattering, and bounce resonance. On one hand, our study clearly demonstrates that radiation belt electrons and protons can undergo resonant interactions with MS waves at the latitudes away from the geomagnetic equator, distinguishing it from most previous studies that focus on MS waves in the equatorial region. On the other hand, the Landau resonance between MS waves and radiation belt electrons has been intensively investigated as a viable candidate of electron acceleration. Our results indicate that cyclotron resonance of MS waves may also be important to affect the dynamics of multi-MeV radiation belt electrons, which should be carefully explored in future studies.

\section{Abbreviation \\ MS waves: magnetosonic waves.}

\section{Authors' contributions}

$X G, B N, W Z, Z X$, and $M H$ have been involved in drafting the manuscript or revising it critically for important intellectual content. $R Z, C Z, X G, W Z$, and JY have made substantial contributions to conception and design, or acquisition of data, or analysis and interpretation of data. XG has given final approval of the version to be published. All authors read and approved the final manuscript.

\section{Acknowledgements}

Thanks to the joint efforts of all my colleagues in the laboratory.

\section{Competing interests}

I confirm that I have read SpringerOpen's guidance on competing interests and have included a statement indicating that none of the authors have any competing interests in the manuscript. 


\section{Availability of data and materials}

Data and materials are available upon request.

\section{Consent for publication}

All the authors agreed to publish the article.

\section{Ethics approval and consent to participate}

This study does not involve human participants and report health-related outcomes.

\section{Funding}

This work was supported by the NSFC Grants 41304130, 41574160, 41474141, and 41204120 and by the Hubei Province Natural Science Excellent Youth Foundation (2016CFA044).

\section{Appendix}

Regarding the 10th-order polynomial equation of resonant frequency, i.e., Eq. (12), its coefficients take the forms below:

$$
\begin{aligned}
& A_{10}=\eta_{9}-2 \eta_{9} b^{2}+\eta_{9} b^{4}, \\
& A_{9}=4 a \eta_{9} b^{2}-4 a \eta_{9}, \\
& A_{8}=6 a^{2} \eta_{9}-\eta_{10}+b^{2} \eta_{13}-2 a^{2} b^{2} \eta_{9}-\eta_{16} b^{4}, \\
& A_{7}=4 a \eta_{10}-4 a^{3} \eta_{9}-2 a \eta_{13} b^{2}, \\
& A_{6}=a^{4} \eta_{9}-6 a^{2} \eta_{10}+\eta_{11}+a^{2} b^{2} \eta_{13}-b^{2} \eta_{14}+b^{4} \eta_{17}, \\
& A_{5}=4 a^{3} \eta_{10}-4 a \eta_{11}+2 a \eta_{14} b^{2}, \\
& A_{4}=6 a^{2} \eta_{11}-a^{4} \eta_{10}-\eta_{12}+b^{2} \eta_{15}-a^{2} b^{2} \eta_{14}-\eta_{18} b^{4}, \\
& A_{3}=4 a \eta_{12}-4 a^{3} \eta_{11}-2 a b^{2} \eta_{15}, \\
& A_{2}=a^{4} \eta_{11}-6 a^{2} \eta_{12}+a^{2} b^{2} \eta_{15}+b^{4} \eta_{19}, \\
& A_{1}=4 a^{3} \eta_{12}, \\
& A_{0}=-a^{4} \eta_{12},
\end{aligned}
$$

where

$$
\begin{aligned}
& \eta_{1}=\Omega_{c i}^{2}+\Omega_{c e}^{2}+\omega_{p i}^{2}+\omega_{p e}^{2}, \\
& \eta_{2}=\Omega_{c i}^{2} \Omega_{c e}^{2}+\omega_{p i}^{2} \Omega_{c e}^{2}+\omega_{p e}^{2} \Omega_{c i}^{2}, \\
& \eta_{3}=\Omega_{c i}^{2}+\Omega_{c e}^{2}, \\
& \eta_{4}=\Omega_{\mathrm{ci}}^{2} \Omega_{\mathrm{ce}}^{2}, \\
& \eta_{5}=\Omega_{c i}^{2}+\Omega_{c e}^{2}+2 \omega_{p i}^{2}+2 \omega_{p e}^{2}, \\
& \eta_{2}=\Omega_{c i}^{2} \Omega_{c e}^{2}+\omega_{p i}^{2} \Omega_{c e}^{2}+\omega_{p e}^{2} \Omega_{c i}^{2},
\end{aligned}
$$

$$
\eta_{7}=\left(\omega_{p e}^{2} \Omega_{c i}-\omega_{p i}^{2} \Omega_{c e}\right)^{2}
$$

$$
\begin{aligned}
& \eta_{8}=\omega_{p i}^{2}+\omega_{p e}^{2}, \\
& \eta_{9}=1+X^{2} \\
& \eta_{10}=\eta_{1} X^{2}+\eta_{3}+\eta_{8}, \\
& \eta_{11}=\eta_{2} X^{2}+\eta_{4}+\eta_{3} \eta_{8}, \\
& \eta_{12}=\eta_{4} \eta_{8} \\
& \eta_{13}=\eta_{5} X^{2}+\eta_{1}\left(2+X^{2}\right)+\eta_{8}\left(2+X^{2}\right), \\
& \eta_{14}=\eta_{6} X^{2}+\eta_{2}\left(2+X^{2}\right)+\eta_{1} \eta_{8}\left(2+X^{2}\right), \\
& \eta_{15}=\eta_{7} X^{2}+\eta_{2} \eta_{8}\left(2+X^{2}\right), \\
& \eta_{16}=\left(\eta_{5}+\eta_{8}\right)\left(1+X^{2}\right), \\
& \eta_{17}=\left(\eta_{6}+\eta_{5} \eta_{8}\right)\left(1+X^{2}\right), \\
& \eta_{18}=\left(\eta_{7}+\eta_{6} \eta_{8}\right)\left(1+X^{2}\right), \\
& a=n \Omega_{c e} / \gamma, \text { and } b=\beta \cos \theta \cos \alpha . \\
& \eta_{8}\left(1+X^{2}\right)
\end{aligned}
$$

\section{Publisher's Note}

Springer Nature remains neutral with regard to jurisdictional claims in published maps and institutional affiliations.

Received: 23 February 2017 Accepted: 29 August 2017

Published online: 12 September 2017

\section{References}

Chang SS, Zhao ZY, Ni BB (2013) Propagation and application of VLF waves generated by HF heating of ionosphere in the magnetosphere. Chinese J Geophys 56(12):3960-3968. doi:10.6038/cjg20131202 (in Chinese)

Chen L, Maldonado A, Bortnik J et al (2015) Nonlinear bounce resonances between magnetosonic waves and equatorially mirroring electrons. J Geophys Res Space Phys 120:6514-6527. doi:10.1002/2015JA021174 Fu S, Ni B, Li J, Zhou C, Gu X, Huang S, Zhang H, Ge Y, Cao X (2016) Interactions between magnetosonic waves and ring current protons: gyroaveraged test particle simulations. J Geophys Res Space Phys 121:8537-8553. doi:1 $0.1002 / 2016 J$ A023117 
Gannon JL, Li X, Heynderickx D (2007) Pitch angle distribution analysis of radiation belt electrons based on combined release and radiation effects satellite medium electrons a data. J Geophys Res 112:A05212. doi:10.102 9/2005JA011565

Gu XD, Zhao ZY, Xiang W et al (2011) Statistical analysis of energetic electron fluxes in the Earth's radiation belts under different geomagnetic activities. Chin J Geophys 54(3):656-665. doi:10.3969/j.issn.0001-5733.2011.03.004 (in Chinese)

Horne RB, Thorne RM, Glauert SA et al (2007) Electron acceleration in the Van Allen radiation belts by fast magnetosonic waves. Geophys Res Lett 34:L17107. doi:10.1029/2007GL030267

Laakso H, Junginger H, Roux A et al (1990) Magnetosonic waves above fc $(\mathrm{H}+)$ at geostationary orbit: GEOS 2 results. J Geophys Res 95(A7):1060910621. doi:10.1029/JA095iA07p10609

Li LY, Cao JB, Zhou CC (2008) Whistler-mode waves modify the high-energy electron slot region and the outer radiation belt. Chine J Geophys 51(2):316-324 (in Chinese)

Li J, Ni B, Xie L et al (2014) Interactions between magnetosonic waves and radiation belt electrons: comparisons of quasi-linear calculations with test particle simulations. Geophys Res Lett 41:4828-4834. doi:10.1002/2 014GL060461

Li J et al (2016a) Formation of energetic electron butterfly distributions by magnetosonic waves via Landau resonance. Geophys Res Lett 43:3009_ 3016. doi:10.1002/2016GL067853

Li J et al (2016b) Ultrarelativistic electron butterfly distributions created by parallel acceleration due to magnetosonic waves. J Geophys Res Space Phys 121:3212-3222. doi:10.1002/2016JA022370

Ma Q, Li W, Thorne RM et al (2013) Global distribution of equatorial magnetosonic waves observed by THEMIS. Geophys Res Lett 40:1895-1901. doi:10.1002/grl.50434

Ma Q, Li W, Thorne RM, Bortnik J, Kletzing CA, Kurth WS, Hospodarsky GB (2016) Electron scattering by magnetosonic waves in the inner magnetosphere. J Geophys Res Space Phys 121:274-285. doi:10.1002/201 5JA021992
Maldonado AA, Chen L, Claudepierre SG, Bortnik J, Thorne RM, Spence H (2016) Electron butterfly distribution modulation by magnetosonic waves. Geophys Res Lett 43:3051-3059. doi:10.1002/2016GL068161 Mourenas D, Artemyev AV, Agapitov OV et al (2013) Analytical estimates of electron quasi-linear diffusion by fast magnetosonic waves. J Geophys Res Space Phys 118:3096-3112. doi:10.1002/jgra.50349

Russell CT, Holzer RE, Smith EJ (1970) OGO 3 observations of ELF noise in the magnetosphere: the nature of equatorial noise. J Geophys Res 75(4):755-768. doi:10.1029/JA075i004p00755

Santolík O, Pickett JS, Gurnett DA et al (2002) Spatiotemporal variability and propagation of equatorial noise observed by cluster. J Geophys Res 107(A12):1495. doi:10.1029/2001JA009159

Sheeley BW, Moldwin MB, Rassoul HK et al (2001) An empirical plasmasphere and trough density model: CRRES observations. J Geophys Res 106(A11):25631-25641. doi:10.1029/2000JA000286

Stix T (1962) The theory of plasma waves. McGraw-Hill, New York

Tsurutani BT, Falkowski BJ, Pickett JS, Verkhoglyadova OP, Santolik O, Lakhina GS (2014) Extremely intense ELF magnetosonic waves: a survey of polar observations. J Geophys Res Space Phys 119(2):964-977

Xiao F, Zong Q, Wang Y et al (2014) Generation of proton aurora by magnetosonic waves. Sci Rep 4:5190. doi:10.1038/srep05190

Xiao F, Yang C, Su Z et al (2015) Wave-driven butterfly distribution of Van Allen belt relativistic electrons. Nat Commun 6:8590. doi:10.1038/ncomms9590

Xie L, Tu WC, Zhang H et al (2007) NOAA/POES observation of the sources of the relativistic electron in the magnetosphere. Chin J Geophys 50(6):1632-1638 (in Chinese)

Zhima Z, Chen L, Fu H, Cao J, Horne RB, Reeves G (2015) Observations of discrete magnetosonic waves off the magnetic equator. Geophys Res Lett 42(22):9694-9701. doi:10.1002/2015GL066255

\section{Submit your manuscript to a SpringerOpen ${ }^{\odot}$ journal and benefit from:}

- Convenient online submission

- Rigorous peer review

- Open access: articles freely available online

- High visibility within the field

- Retaining the copyright to your article

Submit your next manuscript at $\boldsymbol{\nabla}$ springeropen.com 